\title{
A GENUS- AND SPECIES-SPECIFIC NESTED POLYMERASE CHAIN REACTION MALARIA DETECTION ASSAY FOR EPIDEMIOLOGIC STUDIES
}

\author{
BALBIR SINGH, ALBINO BOBOGARE, JANET COX-SINGH, GEORGES SNOUNOU, \\ MOHAMMAD SHUKRI ABDULLAH, AND HASAN ABDUL RAHMAN \\ School of Medical Sciences, Universiti Sains Malaysia, Kubang Kerian, Kelantan, Malaysia; Department of Infection and \\ Tropical Medicine, Imperial College School of Medicine, Northwick Park Hospital, Harrow, United Kingdom; Sabah State \\ Department of Health, Kota Kinabalu, Sabah, Malaysia
}

\begin{abstract}
A nested polymerase chain reaction (PCR) assay that uses Plasmodium genus-specific primers for the initial PCR (nest 1) amplification and either genus- or species-specific primers for the nest 2 amplifications was tested on laboratory and field samples. With in vitro cultured Plasmodium falciparum-infected blood samples, it was capable of detecting six parasites/ $\mu$ l of blood using DNA prepared from 25 - $\mu$ l blood spots on filter paper. The assay was evaluated on fingerprick blood samples collected on filter paper from 129 individuals living in a malaria-endemic area in Malaysia. Malaria prevalence by genus-specific nested PCR was $35.6 \%$ (46 of 129) compared with $28.7 \%$ (37 of 129) by microscopy. The nested PCR detected seven more malaria samples than microscopy in the first round of microscopic examination, malaria in three microscopically negative samples, six double infections identified as single infections by microscopy and one triple infection identified as a double infection by microscopy. The nested PCR assay described is a sensitive technique for collecting accurate malaria epidemiologic data. When coupled with simple blood spot sampling, it is particularly useful for screening communities in remote regions of the world.
\end{abstract}

Microscopy is the method of choice for the diagnosis of malaria in endemic areas because it is an inexpensive and rapid method of detection. Correct identification of the four species of Plasmodium causing human malaria and the level of detection by microscopy depends on a number of factors, including the experience of the microscopist, proper staining of the slides, appropriate maintenance of microscopes, and the time spent examining each slide. At best, the sensitivity of detection by microscopy is approximately 10-30 parasites/ $\mu 1$ of blood. ${ }^{1}$ However, this level of detection is normally not attained in malaria-endemic areas and particularly during epidemiologic studies when many samples need to be screened in a relatively short time. Thus, incorrect speciation is common and mixed infections and low levels of parasitemia may be missed.

To overcome some of the limitations of microscopy for detection of malaria, polymerase chain reaction (PCR)based assays have been developed for the detection and identification of malaria parasites. These methods have proved to be more specific and sensitive than conventional microscopy and some are reported to be able to detect as few as one parasite/ $\mu$ l of blood. ${ }^{2-6}$ However, to attain such a high sensitivity, blood samples collected from individuals have to be processed immediately or stored at low temperatures and the steps involved in DNA template preparation were multistep, often requiring biohazardous chemicals. Since malaria remains a problem of underdeveloped and often remote areas of the world, it is important to couple PCRbased assays with simple sampling and DNA extraction methods to maximize the value of PCR assays. In a previous study, we coupled the nested PCR assay of Snounou and others $^{3}$ to blood collection on filter papers and a simple DNA extraction method and found that the assay was able to detect more single and mixed malaria infections compared with microscopy. ${ }^{7}$ This nested PCR assay involved sequential PCR amplifications for each blood sample screened. In the first PCR (nest 1) amplification, Plasmodium-specific primers were used. The product of this nest 1 amplification then served as DNA template for four separate second PCR (nest
2) amplifications with primers specific for each of the 4 human malaria species. Therefore, to screen a blood sample, a total of five PCR amplifications were required and four PCR products had to be analyzed by gel electrophoresis. For epidemiologic studies, it would be more cost-effective and faster if samples could be initially screened using Plasmodium genus-specific primers in both nest 1 and 2 amplifications and only positive samples subjected to subsequent speciation by further nest 2 amplifications.

In this study we describe a nested PCR assay that detects malaria in blood samples collected on filter paper. It uses Plasmodium genus-specific primers for the nest 1 and nest 2 amplifications and reuses the nest 1 amplification products of positive samples for species-specific nest 2 amplifications. The sensitivity of detection was determined and the assay was evaluated on field samples collected in a malaria-endemic area in Malaysia.

\section{MATERIALS AND METHODS}

Parasite culture. The $P$. falciparum 3D7 clone, donated by Professor D. Walliker (University of Edinburgh, Edinburgh, Scotland) was maintained in continuous culture by the candle jar method of Trager and Jensen. ${ }^{8}$ For sensitivity studies, cultures were synchronized using sorbitol ${ }^{9}$ and allowed to grow for an additional $48 \mathrm{hr}$ before Percoll density centrifugation ${ }^{10}$ was done to remove any late trophozoite or schizont-infected erythrocytes. The resultant blood sample, containing only ring-stage parasites, was serially diluted five-fold using uninfected blood. Aliquots of $25 \mu$ l were spotted directly onto 3 MM chromatography paper (Whatman International Corp., Maidstone, United Kingdom), allowed to air-dry, and stored at room temperature until required.

Study area. Individuals living in Bitoon, a malaria-endemic area located $200 \mathrm{~km}$ northeast of Kota Kinabalu, Sabah, Malaysia, were examined during a malaria survey by officers of the Sabah State Vector-Borne Diseases Control 